\title{
Rheology and Structure of Cornstarch Suspensions in Water-Poly(propylene glycol) Mixtures
}

\author{
Spencer E. Taylor \\ Department of Chemistry, University of Surrey, Guildford, Surrey GU2 7XH, UK
}

\begin{abstract}
$\underline{\text { Abstract }}$
Concentrated aqueous cornstarch (CS) suspensions are often used to demonstrate an extreme example of shear thickening rheological behaviour. Here, we describe the increased rheological complexity that occurs on the addition of poly(propylene glycol) (PPG) to an aqueous CS suspension. The appearance of shear thickening/jamming, shear thinning, yield stress and near-Newtonian behaviours is dependent on the PPG:water ratio. Rheological measurements have been complemented by dielectric measurements and optical microscopy. The complex behaviour is interpreted in terms of reduced electrostatic stabilisation of the CS particles with increased poly(propylene glycol) concentration. The analysis also suggests why cornstarch suspensions in water exhibit particularly good shear thickening characteristics.
\end{abstract}

Keywords: Cornstarch, colloid stability, suspensions, rheology

Running head: Rheology and structure of cornstarch suspensions 


\section{INTRODUCTION}

Suspension rheology is fundamental to the formulation of a wide variety of traditional and hi-tech multiphase products, as well as being a significant consideration for the efficient operation of industrial processes.

In general, increasing the internal phase concentration of particulate suspensions causes an increase in non-Newtonian rheological characteristics, through an increasing dominance of particle interactions controlled by colloidal forces in Brownian systems and hydrodynamic conditions in systems containing non-Brownian particles.

Non-Newtonian effects can generally be defined by the shear conditions (i.e. shear rate, shear stress) under which they appear, viz. yield stress at zero or very low shear; shear thinning at low to moderate shear, and shear thickening at high shear. This order reflects the energetics of the processes responsible for the respective types of behaviour. The yield condition therefore refers to breaking of individual inter-particle contacts; hydrodynamic streamlining leads to particle redistribution resulting in shear thinning; and the formation of so-called hydroclusters has been advanced as one explanation for shear thickening. ${ }^{[1]} \mathrm{A}$ schematic representation of these different conditions is shown in Fig. 1.

\section{[FIGURE 1]}

In the absence of attractive particle interactions, the suspension rheology is more appropriately characterised by Newtonian regions at low and high shear. These are separated by a region of shear thinning which, as depicted in Fig. 1, results from 
streamlining of the particles in the flow. On the other hand, the dashed curve represents the effect of inter-particle attraction, leading to high viscosity at low shear rates, and possibly also indicating a yield stress condition. Increasing the shear leads to structural breakdown, with concomitant viscosity reduction. Also represented in Fig. 1 is a shear thickening region generated by the formation of hydroclusters at still higher shear rates. This can then promote a further Newtonian region which may also shear thin. However, some systems also attain an extreme 'jammed' shear thickened state with an infinite viscosity, as the dispersed particles are confined in a close-packed arrangement.

As observed by Wagner and Brady, ${ }^{[2]}$ shear thickening fluids not only represent scientific curiosities, they also provide industrial challenges and innovation potential. During the past few years a number of potential applications of shear thickening fluids have been identified, including impact-absorbing products, ranging from sportswear, where mild shear thickening responses are appropriate,${ }^{[3]}$ to flexible armour that can withstand knife or bullet penetration; ${ }^{[4]}$ oilfield-related applications, such as gravel packing, ${ }^{[5]}$ drill cuttings removal $^{[6]}$ and conformance control agents ${ }^{[7]}$ oral care products ${ }^{[8]}$ and household ${ }^{[9]}$ cleansing compositions which thicken under stress; and mechanical transmission systems, such as a braking mechanism for restricting the speed of revolving doors. ${ }^{[10]}$

The present investigation was in part inspired by the increasing diversity of applications as well as the recent analysis by Brown et al. ${ }^{[1]}$ which explored the relationship between different rheological responses exhibited by concentrated suspensions, for example treating shear thickening and yield stress as competing effects, which emphasises the respective predominance of repulsive and attractive inter-particle interactions.

We aimed, therefore, to investigate rheological consequences of changing the composition of the dispersion medium, in the shear thickening cornstarch (CS)/water 
system which we know exhibits substantial shear thickening and jamming above a relatively modest particle volume fraction. ${ }^{[12]}$ We envisaged that, since a prerequisite for shear thickening is that the suspension is not aggregated, ${ }^{[13]}$ a system such as CS/water would be reasonably sensitive to changes in solvent polarity, which would modify particle interactions. ${ }^{[14]}$ To this end, we have used mixtures of water and poly(propylene glycol) (PPG) to modulate the solvent characteristics in order to determine concomitant effects on the rheology and microstructure of the suspensions. To the author's knowledge there have been no specific investigations concerning colloidal stability or microstructure of aqueous CS suspensions.

It is reasonable to suppose, however, that CS particles share some common features with starch granules from other sources, and in particular, that they will be negatively charged in water except at very low $\mathrm{pH}$ conditions. Marsh and Waight determined zeta potentials for wheat and potato starch granules, the former showing an isoelectric point $\left(\mathrm{pH}_{\mathrm{iep}}\right)$ of 3.7. ${ }^{[15]}$ This behaviour was attributed to the presence of titratable surface species, including proteins and phospholipids, which provide electrostatic repulsion between the granules in aqueous suspensions; further evidence for electrostatic stabilisation came from the observation that flocculation occurred at the isoelectric point of wheat starch. ${ }^{[15]}$

Thus, it was anticipated that increasing the proportion of PPG to water in the dispersion medium would reduce electrostatic repulsion between CS particles, potentially reducing the shear thickening behaviour, whereupon shear thinning may then be expected to be the dominant rheological feature of the suspensions. However, as will be described, the mixed solvent system was more complex in terms of the range of rheological behaviours observed, and in this respect, complements Brown et al.'s recent analysis. ${ }^{[1]}$ 


\section{EXPERIMENTAL}

\subsection{Materials}

The CS used in this study was a single batch of an additive-free "own brand" product purchased from a leading UK supermarket. It was used as received and stored in a closed container to maintain a constant water level. This product was stated to contain $88 \%$ CS (a minimum value), $0.3 \%$ protein and $0.1 \%$ lipid, the remainder being water, which was determined to be $9.3 \%$ by heating to constant weight at $80^{\circ} \mathrm{C}$. This is a typical composition for cornstarch. ${ }^{[16]}$ The $\mathrm{pH}$ of aqueous suspensions (from 5 to $50 \mathrm{wt} \% \mathrm{CS}$ ) was found to be $4.6 \pm 0.1$, with a $\mathrm{pH}_{\mathrm{iep}}(5 \mathrm{wt} \%$ suspension $)$ of $2.6 \pm 0.2$, determined using a Mütek Particle Charge Detector. PPG (m.w. 425) was purchased from Fisher Scientific and also used as received. Water was deionised. PPG/water mixtures of the required compositions were prepared on a weight basis (NB the densities of water and PPG are almost identical at $20^{\circ} \mathrm{C}$ ) and are subsequently quoted in terms of the PPG mass fraction, ${ }_{15} \phi_{\text {PPG }}$.

\subsection{Methods}

\subsubsection{Preparation of suspensions}

$50 \mathrm{wt} \%$ CS suspensions were identified as being suitable for study since lower concentrations were too fluid for useful measurements to be made at low $\phi_{\mathrm{PPG}}$, while higher CS concentrations were too viscous for higher $\phi_{\mathrm{PPG}}$. The suspensions were prepared by vigorously hand mixing equal weights (typically 15-20 g) of CS and the different PPG/water mixtures using a spatula, until a visually uniform product was obtained. The suspensions were used immediately after preparation. 


\subsubsection{Rheological measurements}

A Carri-Med CSL-50 controlled stress rheometer and $4^{\circ}$ cone-and-plate measuring system were used to determine flow and oscillation rheology of the suspensions. All rheological measurements were made at least in duplicate. Viscosities of the PPG/water mixtures were measured using a Contraves Low Shear 30 rheometer. All measurements were made at $25^{\circ} \mathrm{C}$.

\subsubsection{Microscopic characterisation of particles in suspensions}

The particle size distribution of the CS sample used in the present study was measured using a Visual Process Analyser (ViPA) instrument (Jorin Ltd., Leicester, UK). This is an image analysis-based technique which classifies particles in terms of size and shape. Particle size data is quoted as the average of four Feret diameters taken at $45^{\circ}$ intervals from the image cross-section. Suitable CS samples were prepared by dilution of concentrated aqueous suspensions. The instrument requires a flow rate of $\approx 25 \mathrm{~mL} / \mathrm{min}$ which ensures the collection of undistorted particle images, thereby avoiding multiple counting.

For the purposes of microscopic examination, the original $50 \mathrm{wt} \% \mathrm{CS}$ suspensions were diluted precisely to $1 \mathrm{wt} \%$. As a result of dilution and subsequent application of small ${ }_{20}$ aliquots to uncovered glass microscope slides, the samples will inevitably be subjected to a degree of shearing, but they should retain evidence of the original microstructure. Still images and movie clips were analysed using a Motic digital imaging system and Motec Images 2.0 ML software to visualise aggregation and produce aggregation number information for different $\phi_{\mathrm{PPG}}$. 


\subsubsection{Dielectric spectroscopy}

Dielectric spectra of the suspensions were determined for the frequency range $5 \times 10^{-2}$ to $2 \times 10^{7} \mathrm{~Hz}$ using a Novocontrol broadband dielectric spectrometer (Novocontrol Technologies $\mathrm{GmbH}$, Hundsangen, Germany) at ambient temperature $\left(23.0 \pm 1.0^{\circ} \mathrm{C}\right)$. Since the composition of the dispersion medium is the principal variable, the dielectric parameters are expressed as ratios of the suspension properties relative to the specific dispersion media at each frequency.

\subsubsection{Physical characterisation of PPG/water mixtures}

Each PPG/water mixture was characterised in terms of its surface tension and dielectric constant in view of the relevance of these properties to the formation and stabilisation of suspensions. ${ }^{[17]}$ Surface tensions were determined at $23.0 \pm 1.0^{\circ} \mathrm{C}$ by the du Nouy ring method using a Krüss K10 tensiometer. The Pt ring was acid-washed, water-rinsed and ${ }_{15}$ heated to redness in a blue Bunsen flame before each measurement. The Novocontrol system described above was used to determine dielectric constants of the mixtures at ambient temperature from high frequency permittivity measurements after previously calibrating with fluids of known dielectric constant.

\section{RESULTS}

\subsection{Macroscopic and microscopic characterisation}

Fig. 2 show photographs of $50 \mathrm{wt} \% \mathrm{CS}$ suspensions prepared from the various PPG/water mixtures. In water and at low PPG concentrations $\left(\phi_{\mathrm{PPG}}<0.2\right)$, the CS dispersions are fluid at rest but noticeably shear thickening when stirred. At higher PPG 
concentrations, the suspensions at rest become increasingly more structured, being selfsupporting, resembling whipped cream, and losing their obvious shear thickening characteristics. The $100 \%$ PPG suspension flows readily (Fig. 2).

\section{[FIGURE 2]}

The above structural differences are also evident from the microscopic examination of dilute (1 wt $)$ suspensions shown in Fig. 3. In water, the CS particles are well dispersed; micrographs for $\phi_{\mathrm{PPG}}=0.1$ and 0.2 contain signs of particle chains. At $\phi_{\mathrm{PPG}}=0.5$, more substantial aggregated particle chains provide evidence for stronger aggregation, consistent with the highly structured suspension exhibiting a yield stress shown in Fig. 2. At the two highest $\phi_{\mathrm{PPG}}$ suspensions, the particle chains are absent, being replaced by dispersed clusters of varying size. Quantitative analysis of images for the suspensions shown in Fig. 3 generates the particle cluster size distributions shown in Fig. 4, showing the cumulative percentages of particles in clusters of the indicated size. This analysis shows that the median aggregation number (i.e. corresponding to $50 \%$ of the particles) is approximately constant below $\phi_{\mathrm{PPG}}=0.2$ (the majority being single particles), but increases significantly, to an aggregation number of $\approx 10$ at $\phi_{\mathrm{PPG}}=0.5$. The distribution median almost doubles for $\phi_{\mathrm{PPG}}=0.8$, before decreasing to $<10$ for the PPG suspension.

\section{[FIGURE 3]}

These results are broadly in line with expectation, based on the appearance of the suspensions, although the analysis makes no distinction between the morphology of the 
aggregates (e.g. whether linear chains or globular clusters, examples of both being seen in Fig. 2).

\section{[FIGURE 4]}

Fig. 5 shows number and volume particle size distributions for water and PPG-based CS suspensions. The log-normal number distributions are essentially monomodal, with mean diameters $\left(d_{1,0}\right)$ of 13.3 and $13.8 \mu \mathrm{m}$ in water and PPG, respectively, therefore indicating the absence of swelling, and consistent with other reported CS size data. ${ }^{[12,18]}$ However, the PPG volume distribution shows evidence of particle aggregation, which is also reflected in the volume mean diameters $\left(d_{4,3}\right)$ in water and PPG of 18.7 and $32.9 \mu \mathrm{m}$, respectively.

\section{[FIGURE 5]}

\subsection{Rheological characterisation}

Flow curves for the suspensions are collected in Fig. 6, plotted as viscosity ratio as a function of shear rate. The viscosity ratio, $\eta_{r}=\eta_{s} / \eta_{0}$, where $\eta_{s}$ is the shear ratedependent apparent viscosity of the suspension and $\eta_{0}$ is the Newtonian viscosity of the dispersion medium, allows for the effect of changing the composition of the latter.

${ }_{20}$ Corresponding dynamic viscosity data from oscillatory measurements are shown in Fig. 7, which also contains comparative data for a higher CS concentration.

[FIGURE 6] 
The shear flow and dynamic viscosity data are characterised by transitions from shear thickening to shear thinning at $\approx 10 \mathrm{~s}^{-1}$ or $5 \mathrm{~Hz}$, respectively, for $\phi_{\mathrm{PPG}}<0.2$. Shear thickening results in an order of magnitude increase in the viscosity parameters in the higher shear rate/frequency ranges. Shear thinning at the lower shear rates is a characteristic of all the suspensions except for $\phi_{\mathrm{PPG}}=1$, which exhibits near-Newtonian behaviour.

\section{[FIGURE 7]}

From Figs. 6 and 7 it is apparent that for $\phi_{\mathrm{PPG}}>0.2$, shear thickening transitions are masked by the presence of yield stress contributions to the resultant shear stress, as observed previously by Brown et al. ${ }^{[11]}$ These workers treated their suspensions as Herschel-Bulkley fluids, in order to derive yield stress contributions to the overall rheology. In the present study, however, we have found that the data are described adequately using the simplified Bingham equation

$$
\tau_{B}=\tau_{0}+\eta_{p l} \dot{\gamma}
$$

where $\tau_{B}$ is the Bingham shear stress, $\tau_{0}$ the yield stress and $\eta_{p l}$ the plastic viscosity. Rearranging Eq. (1) leads to

$$
\eta=\frac{\gamma_{p l}}{\left(1-\tau_{0} / \tau_{B}\right)}
$$

${ }_{20} \quad$ on the basis of which $\eta \square-\tau_{B}$ plots allow $\eta_{p l}$ and $\tau_{0}$ to be determined for different PPG/water mixtures. The resultant Bingham parameters are shown as a function of $\phi_{\mathrm{PPG}}$ in Fig. 8. Both $\eta_{p l}$ and $\tau_{0}$ initially increase with increasing $\phi_{\mathrm{PPG}}$ to reach maximum values in the range $0.5 \leq \phi_{\mathrm{PPG}} \leq 0.8$, corresponding to the suspensions being at their most structured 
under low shear rate conditions (Fig. 2), as exemplified by the flow-packed arrangement shown in the inset in Fig. 6 . Above $\phi_{\mathrm{PPG}}=0.8$, a decrease in both parameters is consistent with the observed loss of structure.

[FIGURE 8]

\subsection{Dielectric characterisation}

Both the permittivity and conductivity results (each expressed as the ratio of the suspension value to the solvent value at each frequency) indicate a transition in behaviour occurring at $\phi_{\mathrm{PPG}} \approx 0.2$. Thus, permittivity ratio and conductivity ratio spectra for different PPG/water mixtures are given in Fig. 9. For $\phi_{\mathrm{PPG}}<0.2$, the permittivity of the suspension exceeds that of the dispersion medium by up to two orders of magnitude. On the other hand, for $\phi_{\mathrm{PPG}}>0.2$, the suspension permittivities are reduced by up to two orders of magnitude.

\section{[FIGURE 9]}

At low $\phi_{\mathrm{PPG}}$, the permittivity ratio spectra exhibit maxima in the range $10^{3}-10^{4} \mathrm{~Hz}$, whereas $\phi_{\mathrm{PPG}^{-}}$-dependent minima in the $10^{1}-10^{3} \mathrm{~Hz}$ range are more characteristic of the higher $\phi_{\mathrm{PPG}}$ suspensions. For pure PPG, the spectrum is more complex, exhibiting both a small maximum $\left(\right.$ at $\left.\approx 10^{2} \mathrm{~Hz}\right)$ and minimum $\left(\right.$ at $\left.\approx 10^{-1} \mathrm{~Hz}\right)$ values. For $\phi_{\mathrm{PPG}}<0.2$, suspension conductivity is up to an order of magnitude greater than for the solvent alone, 
whereas for $\phi_{\mathrm{PPG}}>0.2$, conduction is significantly reduced. Once again, for pure PPG the behaviour is more complex, with both types of behaviour being apparent.

Although a rigorous analysis of the dielectric results is beyond the scope of the present paper, these findings provide information relating to the relative conductivities of components in the suspensions, as will be discussed later. In this respect, to simplify the analysis, Fig. 10 contains conductivity ratio data at a single frequency $(1 \mathrm{kHz})$ as a function of $\phi_{\mathrm{PPG}}$.

[FIGURE 10]

\subsection{Surface tension of PPG/water mixtures}

The effect of PPG/water composition on the equilibrium surface tension of PPG/water mixtures is shown in Fig. 11. Although low m.w. PPGs are known to be less active at the water surface than their higher m.w. counterparts, ${ }^{[19]}$ the surface tension of PPG/water mixtures approaches the PPG value for $\phi_{\mathrm{PPG}}>0.2$, the results being in good agreement with available low $\phi_{\mathrm{PPG}}$ literature data, ${ }^{[20]}$ and are well described by Eq. (3) developed for aqueous-organic mixtures by Connors and Wright; ${ }^{[21]}$ in the present system, $\gamma_{W}$ and $\gamma_{P P G}$ are the respective surface tensions of water and PPG, $x_{W}$ and $x_{P P G}$, their respective mole fractions in the mixture, and $a$ and $b$ are empirical constants. For this system, at $23{ }^{\circ} \mathrm{C}: \gamma_{W}$ ${ }_{20} \quad=71.5 \mathrm{mN} \mathrm{m}^{-1} ; \gamma_{P P G}=32.3 \mathrm{mN} \mathrm{m}^{-1} ; a=0.999 ; b=0.926$.

$$
\gamma=\gamma_{W}-\left(1+\frac{b x_{W}}{\left(1-a x_{W}\right)}\right) x_{P P G}\left(\gamma_{W}-\gamma_{P P G}\right)
$$


[FIGURE 11]

\section{DISCUSSION}

\subsection{Structure and surface properties of starch}

Before discussing the results of this study, it is worth considering the structural features of CS particles of relevance to their dispersion properties. Starch is produced naturally in plant endosperms as water-insoluble granules, built up from alternating semi-crystalline and amorphous layers, each containing different proportions of the $\alpha$-glucan biopolymers amylose and amylopectin. ${ }^{[22,23]}$ Associated with the basic starch granule structure are minor non-starch lipid and protein components, a significant fraction of which will influence the surface and interfacial properties of the granule, and in turn influence the properties of the granule itself. ${ }^{[2]}$ For example, it is found that the surface lipids help to protect against starch gelatinisation in water. ${ }^{[25]}$

The isoelectric point, $\mathrm{pH}_{\mathrm{iep}}$, of the $\mathrm{CS}$ used in this study was found to be 2.6, indicating that the particles will be negatively charged when dispersed in water. By way of comparison, the $\mathrm{pH}_{\mathrm{iep}}$ for wheat starch in water has been reported to be $3.7,{ }^{[15]}$ the ${ }_{20}$ corresponding zeta potential at $\mathrm{pH} 6$ and above being $\approx-30 \mathrm{mV}$, which was ascribed to ionisation equilibria involving surface proteins and phospholipids. ${ }^{[15]}$

\subsection{Suspension stability and interactions in mixed media}


The present results show that the CS suspensions display a considerable dependence on the composition of the PPG/water mixtures. The addition of hydrophobic solutes to water induces considerable structural changes in the vicinity of the bulk solute molecules, through hydrophobic hydration - so-called "iceberg formation", ${ }^{[26]}$ consequences of which are also evident in the surface properties, notably the surface excess and surface tension. ${ }^{[27]}$ Although relevant literature is scarce, it seems reasonable to assume that the suspension properties will be in some way related to the surface tension. In one recent paper, Elblbesy and Hereba identified that erythrocyte cell-cell adhesion increased with decreasing surface tension of the suspending medium. ${ }^{[28]}$ Therefore, on the basis of Fig. 11, it would be expected that the major changes involving surface structuring and hydrophobic hydration would be complete by $\phi_{\mathrm{PPG}} \approx 0.2$, by which point it is seen that shear thickening rheology has given way to a yield stress condition (Figs. 6 and 8).

However, as outlined earlier, other factors are also important. In water, the CS particles will be charged as a result of dissociation of surface groups, and will therefore generate electric double layer repulsion between the starch particle surfaces. This contributes to the colloidal stability of the CS suspensions required for shear thickening. ${ }^{[13]}$

It is also well known that changes in the dielectric constant of mixed aqueous/organic media produce associated effects on the dispersion force and the composition of the electric double layer. ${ }^{[29]}$ Seebergh and Berg summarise the main effects for particle-particle interactions based on alcohol/water mixtures, ${ }^{[30]}$ and even though we have used a more hydrophobic cosolvent, some features are relevant to the present study:

(a) The presence of increasing organic component concentrations reduces the dielectric constant of the medium. This is shown for the PPG/water mixtures in Fig. 12. In turn, this 
reduces the charge distribution near a planar surface, according to the appropriate solution of the Poisson-Boltzmann equation. For small surface potentials,

$$
\Phi=\Phi_{0} \exp (-\kappa x)
$$

where $\Phi_{0}$ and $\Phi$ are the respective electrical potentials at the surface and a distance $x$ from the surface. For a symmetrical electrolyte of ionic charge $z, n_{0}$ is the number concentration of each ion, $e$ is the electronic charge and $k$ the Boltzmann constant, the double layer thickness, $\square \kappa^{-1}$, is given by

$$
\kappa^{-1}=\left(\frac{\varepsilon \varepsilon_{0} k T}{2 n_{0} z e}\right)^{1 / 2}
$$

From Eq. (5), $\kappa \square^{-1} \propto \mathcal{E}^{1 / 2}$, with all other factors remaining constant, and experimentally the decrease in $\kappa \square^{-1}$ in alcohol/water mixtures has been correlated with a reduction in ${ }_{15}$ stability of some colloidal systems. ${ }^{[14,29,31]}$ However, there are also exceptions to this, and so the situation is far from being clear-cut. For example, lower alcohol concentrations have also been found to increase the absolute zeta potential, a finding interpreted in terms of the adsorption of alcohol to the particle surface with concomitant displacement of counterions from the Stern layer into the diffuse layer. ${ }^{[32]}$ In the same way, PPG adsorption at the CS $20 \quad$ interface would also be expected to modify double layer structure.

\section{[FIGURE 12]}

(b) Solvent composition governs the interaction between dispersed particles through its effect on the Hamaker constant, $A_{121}$. The van der Waals attraction energy between two 
similar surfaces (designated by subscript 1) immersed in a medium (subscript 2) and separated by a distance $D$ is given by ${ }^{[33]}$

$$
U_{A}=-\frac{A_{121}}{12 \pi D^{2}} \text { per unit area. }
$$

Hamaker constants for the liquid media can be approximated according to the equation $^{[33]}$

$$
A_{22}=\frac{3}{4} k T\left(\frac{\varepsilon-1}{\varepsilon+1}\right)^{2}+\frac{3 h v_{e}}{16 \sqrt{2}} \cdot \frac{\left(n^{2}-1\right)^{2}}{\left(n^{2}+1\right)^{3 / 2}}
$$

in conjunction with the dielectric constant values of 78.5 and 8.71 (Fig. 12) and refractive index $(n)$ values of 1.333 and 1.447 , for water and $\mathrm{PPG},{ }^{[34]}$ respectively. The absorption frequency, $v_{e}$, was assumed to be constant at $\approx 3.0 \times 10^{15} \mathrm{~s}^{-1}$ following Israelachvili. ${ }^{[34]}$ The calculated $A_{22}$ values are $3.73 \times 10^{-20}$ and $5.99 \times 10^{-20} \mathrm{~J}$, respectively. The present author has been unable to find Hamaker constant data for CS in the literature, but from adhesion force measurements, Shimada et al. estimated $A_{11}$ for potato starch to be $1.5-4.8 \times 10^{-20}$ $\mathrm{J}^{[35]}$ Therefore, for indicative purposes only, $A_{121}$ values for potato starch in water and in PPG are calculated using the combining rule ${ }^{[36]}$

$$
A_{121}=\left(A_{11}{ }^{1 / 2}-A_{22}{ }^{1 / 2}\right)^{2}
$$


to give the values $0.02 \times 10^{-20}$ and $0.45 \times 10^{-20} \mathrm{~J}$, respectively, using the mid-range value for $A_{11}$. Thus, the attraction between starch granules is greater in PPG than in water. Intermediate behaviour would clearly be expected for PPG/water mixtures. ${ }^{[37]}$

(c) In general, the addition of organic solutes to aqueous colloidal dispersions has been observed to reduce the surface charge density, and hence $\Phi_{0}{ }^{\left[{ }^{[2]}\right.}$ This has been explained in terms of cosolvent adsorption at the particle surface which inhibits the dissociation of surface groups. ${ }^{[38]}$ Put another way, reduced water activity in the presence of $\mathrm{PPG}^{[39]}$ would be expected to inhibit dissociation.

A recent atomic force microscopy study directly probed the interaction forces between silica surfaces in 2-propanol/water mixtures, and found direct evidence for each of the above effects. ${ }^{[40]}$ In the case of hydrophilic silica, for example, electric double layer repulsive forces are dominant up to the addition of $10 \%$ 2-propanol. Higher alcohol concentrations caused the forces to become "subdued", until at 100\% 2-propanol, only the van der Waals attraction force was detected. ${ }^{[40]}$

\subsection{Rheological and microstructural properties in PPG/water mixtures}

The present microscopic observations and rheological behaviour are consistent with a reduction in repulsive interactions between particles with increasing $\phi_{\mathrm{PPG}}$.

As discussed above, inter-particle interactions are modulated in the presence of PPG, largely through changes in the dielectric properties of the dispersion medium. In previous studies, temperature or external magnetic and electrical fields have been used to modify inter-particle interactions. ${ }^{[11]}$

As $\phi_{\mathrm{PPG}}$ increases, several features are evident from the steady shear flow curves in Fig. 6. Firstly, the viscosity ratio at low shear rate increases by over an order of magnitude up 
to $\phi_{\mathrm{PPG}}=0.5$, after which it decreases. Similar behaviour is also seen in the dynamic viscosity results in Fig. 7, although in this case no correction is applied for the changing viscosity of the dispersion medium. The second feature is the reduction in the shear thickening transition in the shear rate range $\approx 1-10 \mathrm{~s}^{-1}$ with increasing $\phi_{\mathrm{PPG}}$. Below this shear rate range, the suspensions are shear thinning, with the exception of $100 \%$ PPG which is approximately Newtonian. Following the analysis given by Brown et al., ${ }^{[1]}$ the differences seen in the flow curves are consistent with increasing yield stress contributions. The Bingham yield stress and plastic viscosity data (Fig. 8) are seen to be highly dependent on $\phi_{\mathrm{PPG}}$, which accounts for the range of behaviour observed.

For $\mathrm{pH}$ values above the isoelectric point of $\mathrm{CS}$, suspensions in water will be stabilised by electric double layer repulsion generated by dissociation of surface proteins and phospholipids. At rest, this situation approximates to the equilibrium situation depicted in Fig. 1. As the shear is increased, organisation of the particles in the flow will result in shear thinning. ${ }^{[2]}$ At still higher shear rates, hydrodynamic forces dominate the double layer repulsion which results in the formation of hydroclusters, which impede particle flow leading to shear thickening.

As outlined earlier, the addition of PPG reduces double layer repulsion with consequent effects on the microstructure (Fig. 3) which are clearly evident in the rheology. Thus, as $\phi_{\mathrm{PPG}}$ increases, increasing particle-particle attraction results in the build up of network structures under low shear conditions (Fig. 1). The strength of the networks is evident from the physical appearance of the suspensions (Fig. 2) and is quantified by the Bingham parameters in Fig. 8.

The Bingham yield stress has been related to the energy required to separate individual particle-particle contacts in the structure and the number of such contacts (co-ordination 
number) for each particle. ${ }^{[41]}$ It is not unreasonable to suppose that changes in $\phi_{\mathrm{PPG}}$ will influence both of the latter structural features, the latter varying from zero for individual particles, 2 for open networks comprising chain structures $\left(\phi_{\mathrm{PPG}}<0.2\right)$, and between 8 and 12 for close-packed arrangements, ${ }^{[41]}$ consistent with the appearance and observed microstructure of the suspensions (Figs. 2 and 3). Significantly, the corresponding suspension rheology thereby changes from shear thickening (characteristic of water alone) through an arrested flow (yield stress) condition (approximately $0.2<\phi_{\mathrm{PPG}}<0.8$ ), to an unretarded flow situation (typical of $100 \%$ PPG).

The conductivity ratio results in Fig. 9 shed further light on the properties of the CS suspensions. In particular, it can be shown that the results are consistent with changes in the relative conductivities of CS particles and the dispersion medium. This interpretation takes advantage of the recent analysis of the conductivity of disperse systems comprising particles in a matrix given by Pal. ${ }^{[42]}$ This treatment extends previous theories, e.g. Bruggeman ${ }^{[43]}$ by the inclusion of a maximum packing condition; this serves to distinguish suspensions of solid particles from deformable phases, such as droplets in emulsions or gas bubbles in foams. Pal formulated a general equation that takes into account the conductivities of each phase. Thus, if $\sigma, \sigma_{d}$ and $\sigma_{m}$ are the respective conductivities of the suspension, disperse phase and dispersion medium, then

$$
\left(\frac{\sigma}{\sigma_{m}}\right)^{1 / 3}\left(\frac{\sigma_{d}-\sigma_{m}}{\sigma_{d}-\sigma}\right)=\left(1-\frac{\phi}{\phi_{0}}\right)^{-\alpha \phi_{0}}
$$

where $\phi$ and $\phi_{0}$ are the disperse phase volume fraction and the maximum packing fraction, respectively, and $\square \alpha$ is a near-unity correction factor. If $\lambda$ is the ratio of disperse to continuous phases conductivities $\left(=\sigma_{d} / \sigma_{m}\right), R$ is the ratio of suspension to continuous phase conductivities $\left(=\sigma / \sigma_{m}\right)$, and $\alpha$ is assumed to be unity, then Eq. (9) becomes 


$$
R^{1 / 3}\left(\frac{\lambda-1}{\lambda-R}\right)=\left(1-\frac{\phi}{\phi_{0}}\right)^{-\phi_{0}}
$$

For the CS volume fraction used in the present study (50 wt\% CS in PPG/water mixtures is equivalent to a volume fraction of 0.392 , based on a CS density of $1.5 \mathrm{~g} \mathrm{~cm}^{-3}$ [44] $)$ Eq. (11) allows $\lambda$ to be calculated for each $\phi_{\mathrm{PPG}}$, assuming the term on the right-hand side is assumed to be constant. Illustrative results are shown in Fig. 12 using $\phi_{0}=0.46$, from which it can be seen that with increasing $\phi_{\mathrm{PPG}}, \lambda$ decreases rapidly from its initial high value in water, consistent with a decrease in ionisation of the CS particles.

\section{[FIGURE 12]}

Thus, a reduction in double layer repulsion would also be expected to follow the decrease in CS particle conductivity, which would, in turn, be accompanied by increased particle attraction; in the low $\phi_{\mathrm{PPG}}$ range this leads to relatively weak aggregation as shown in Fig. 3, but at higher PPG concentrations evidence for stronger aggregation is evident. This is also manifest in the rheological results in Figs. 6 - 8. The modification of interparticle forces reduces the stability of the suspensions, with concomitant increases in the low shear viscosity and yield stress of the suspensions, reminiscent of the effect of $\mathrm{pH}$ on yield stress in mineral suspensions in which $\tau_{0}$ is a maximum at the isoelectric point. ${ }^{[45]}$

\subsection{Non-equilibrium phase diagram for CS/PPG/water system}


For the $50 \% \mathrm{CS} /$ water suspension, shear thickening behaviour appears at $\approx 10 \mathrm{~s}^{-1}$ in the steady shear rheology shown in Fig. 6. Increasing $\phi_{\mathrm{PPG}}$ causes the shear thickening discontinuity to shift gradually to lower shear rates, such that by $\phi_{\mathrm{PPG}}=0.2$, the onset occurs at $\approx 1 \mathrm{~s}^{-1}$; thereafter no viscosity increases are evident with increasing shear rate. Brown et al. identified and reported on this type of observation, in which they adopted an analysis that enabled the construction of non-equilibrium phase diagrams describing the different rheological behaviour. ${ }^{[11]}$ In their analysis, the overall shear stress $(\tau)$ was assumed to be made up from shear stress contributions from the different rheological mechanisms. ${ }^{[11]}$ We have applied the same principle to the analysis of our data, i.e.

$$
\tau=\tau_{B}+\tau_{\text {thickening }}+\tau_{\text {Newtonian }}
$$

enabling the contributions from Bingham (Eq. (2)), shear thickening $\left(\tau_{\text {thickening }}\right)$ and Newtonian $\left(\tau_{\text {Newtonian }}\right)$ behaviour to be extracted. Therefore, Fig. 13 shows the nonequilibrium phase diagram based on the present results in which the onset of shear thickening, the upper-bound for shear thickening (including jamming), and yield stress components have been mapped. Intervening regions define the predominance of shear thinning and/or Newtonian behaviour as a function of $\phi_{\mathrm{PPG}}$. On the basis of this analysis, the system is seen to be characterised by a narrow shear thinning region sandwiched between shear thickening (low $\phi_{\mathrm{PPG}}$ ) and yield stress (higher $\phi_{\mathrm{PPG}}$ ) regions. Outside the shear stress- $\phi_{\mathrm{PPG}}$ space, the behaviour is predominantly shear thinning, although the ${ }_{20} \quad$ rheology of the $\square \mathrm{CS} / \mathrm{PPG}$ suspension is surprisingly near-Newtonian.

[FIGURE 13]

\section{CONCLUSIONS}


When poly(propylene glycol) (PPG) is introduced into concentrated aqueous cornstarch (CS) suspensions, the familiar shear thickening behaviour associated with this system changes considerably. Various experimental approaches have been used to analyse the microstructural and rheological properties of the ternary CS/PPG/water system. The study identified various factors that are important in determining the overall properties of the CS suspensions, and an attempt has been made to rationalise the results in terms of the effect of PPG on interactions between CS particles. $50 \mathrm{wt} \% \mathrm{CS}$ suspensions in water and low PPG concentrations exhibit shear thickening as a result of electrostatic stabilisation of the particles owing to dissociation of surface groups. As the PPG concentration is further increased, the concomitant decrease in the dielectric constant of the medium reduces surface dissociation and particle conductivity, thereby also reducing electrostatic repulsion between particle surfaces. This leads to increased particle-particle aggregation, which is manifested rheologically as increased shear thinning and yield stress contributions. The behaviour of the $50 \mathrm{wt} \% \mathrm{CS} / \mathrm{PPG}$ suspension is comparatively unusual in that the rheology becomes almost Newtonian.

\section{Notes and acknowledgements}

The author gratefully acknowledges the award of a Visiting Readership and provision of laboratory facilities by the University of Surrey.

Address correspondence to Dr Spencer Taylor, Department of Chemistry, Faculty of Engineering and Physical Sciences, University of Surrey, Guildford, Surrey, GU2 7XH, UK. E-mail: $\underline{\text { s.taylor@surrey.ac.uk }}$ 


\section{References}

[1] Quemada, D. (1998) Eur. Phys. J. - Appl. Phys., 3: 309-320.

5 [2] Wagner, N.J. and Brady, J.F (2009) Physics Today, 62: 27-32.

[3] Lammer, H., Rosenkranz, H., Kotze, J., and Schwenger, R. (2009) US Patent Appl. 2009/0191989 A1.

[4] Wagner, N. J. and Wenzel, E. D. (2007) US Patent 7226878; Lee, Y.S., Wenzel, E. D., and Wagner, N. J. (2003) J. Mater. Sci., 38: 2825-2833.

${ }_{10}^{[5] \quad Y e h, ~ C . ~ S . ~(1991) ~ U S ~ P a t e n t ~} 5076359$.

[6] Savins, J. G. (1985) US Patent 4496012.

[7] Swanson, B. L. (1980) US Patent 4212747.

[8] Grez, J. and Schallig, M. A. A. (2010) US Patent Appl. 2010/0310301.

[9] Motyka, A. (1996) US Patent 5536437.

${ }_{15}$ [10] Bung, R., Distler, D., and Welzel, G. (1983) German Patent DE 3126841.

[11] Brown, E., Forman, N. A., Orellana, C. S., Zhang, H., Maynor, B. W., Betts, D. E., DeSimone, J. M., and Jaeger, H. M. (2010) Nature Materials, 9: 220-224.

[12] Fall, A., Huang, N., Bertrand, F., Ovarlez, G., and Bonn, D. (2008) Phys. Rev. Lett., 100: 018301.

${ }_{20}$ [13] Gopalakrishnan, V. and Zukoski, C. F. (2004) J. Rheol., 48: 1321-1344.

[14] Odriozola, G., Schmitt, A., Callejas-Fernández, J., and Hidalgo-Álvarez, R. (2007) J. Coll. Interface Sci., 310: 471-480.

[15] Marsh, R. A. and Waight, S. G. (1982) Starch, 34: 149-152. 
[16] White, P. J. (1994) In Specialty Corns, edited by A. R. Hallauer; Boca Raton, FL, CRC Press Inc., p 29.

[17] Kunz, W., Nostro, P. L., and Ninham, B.W. (2004) Curr. Opinion Coll. Interface Sci., 9: $1-18$.

${ }_{5}$ [18] Tester, R. F., Karkalas, J., and Qi, X. (2004) J. Cereal Sci., 39: 151-165.

[19] Tan, S. N., Pugh, R. J., Fornasiero, D., Sedev, R., and Ralston, J. (2005) Min. Eng., 18: 179-188.

[20] Derkaoui, N., Said, S., Grohens, Y., Olier, R., and Privat, M. (2007) J. Coll. Interface Sci., 305: 330-338.

${ }_{10}$ [21] Connors, K. A. and Wright, J. L. (1989) Anal. Chem., 61: 194-198.

[22] Svihus, B., Uhlen, A.K., and Harstad, O.M. (2005) Animal Feed Sci. Technol., 122: 303320.

[23] Buléon, A., Colonna, P., Planchot, V., and Ball, S. (1998) Int. J. Biol. Macromol., 23: 85112.

${ }_{15}$ [24] Baldwin, P. M., Melia, C. D., and Davies, M. C. (1997) J. Cereal Sci., 26: 329-346.

[25] Debet, M. R. and Gidley, M. J. (2006) Carbohydrate Polym., 64: 452-465.

[26] Shinoda, K. (1992) Adv. Coll. Interface Sci., 41: 81-100.

[27] Yano, Y. F. (2005) J. Coll. Interface Sci., 284: 255-259.

[28] Elblbesy, M. and Hereba, A. (2009) Curr. Applied Phys., 9: 872-874.

${ }_{20}$ [29] Lyklema, J. (1976) Pure Appl. Chem., 48: 449-455.

[30] Seebergh, J. E. and Berg, J.C. (1997) Coll. Surf., 121: 89-98.

[31] Ren, J., Song, S., Lopez-Valdivieso, A., Shen, J., and Luz, S. (2001) J. Coll. Interface Sci., 238: 279-284.

[32] Vincent, B. (1992) Adv. Coll. Interface Sci., 42: 279-302. 
[33] Israelachvili, J. (1985) Intermolecular and Surface Forces; Academic Press, London.

[34] http://www.dow.com/polyglycols/ppgc/na/products/ppgs.htm, accessed 6 May 2012.

[35] Shimada, Y., Yonezawa, Y., Sunada, H., Nonaka, R., Katou, K., and Morishita, H. (2000) KONA Powder Particle J., 20: 223-230.

s [36] Hamaker, H. C. (1937) Physica, 4: 1058-1072.

[37] Vincent, B. (1973) J. Coll. Interface Sci., 42: 270-285.

[38] Ottewill, R. H. and Vincent, B. (1972) JCS Faraday Trans. I, 68: 1533-1543.

[39] Sadeghi, R. and Ziamajidi, F. (2006) Fluid Phase Equil., 249: 165-172.

[40] Hupka, L., Nalaskowski, J., and Miller, J. D. (2010) Langmuir, 26: 2200-2208.

${ }_{10}$ [41] Tadros, Th. F. (1987) In Future Directions in Polymer Colloids, edited by M. S. ElAasser and R. M. Fitch; Nijhoff, Dordrecht, p.167.

[42] Pal, R. (2008) J. Composite Mat., 42: 413-425.

[43] Bruggeman, D. A. G. (1935) Ann. Phys. (Leipzig), 24, 636-664.

[44] Pérez, S. and Bertoft, E. (2010) Starch, 62: 389-420.

${ }_{15}$ [45] de Krester, R. G. and Scales, P. J. (2008) J. Coll. Interface Sci., 328: 187-193. 
Figures and Captions

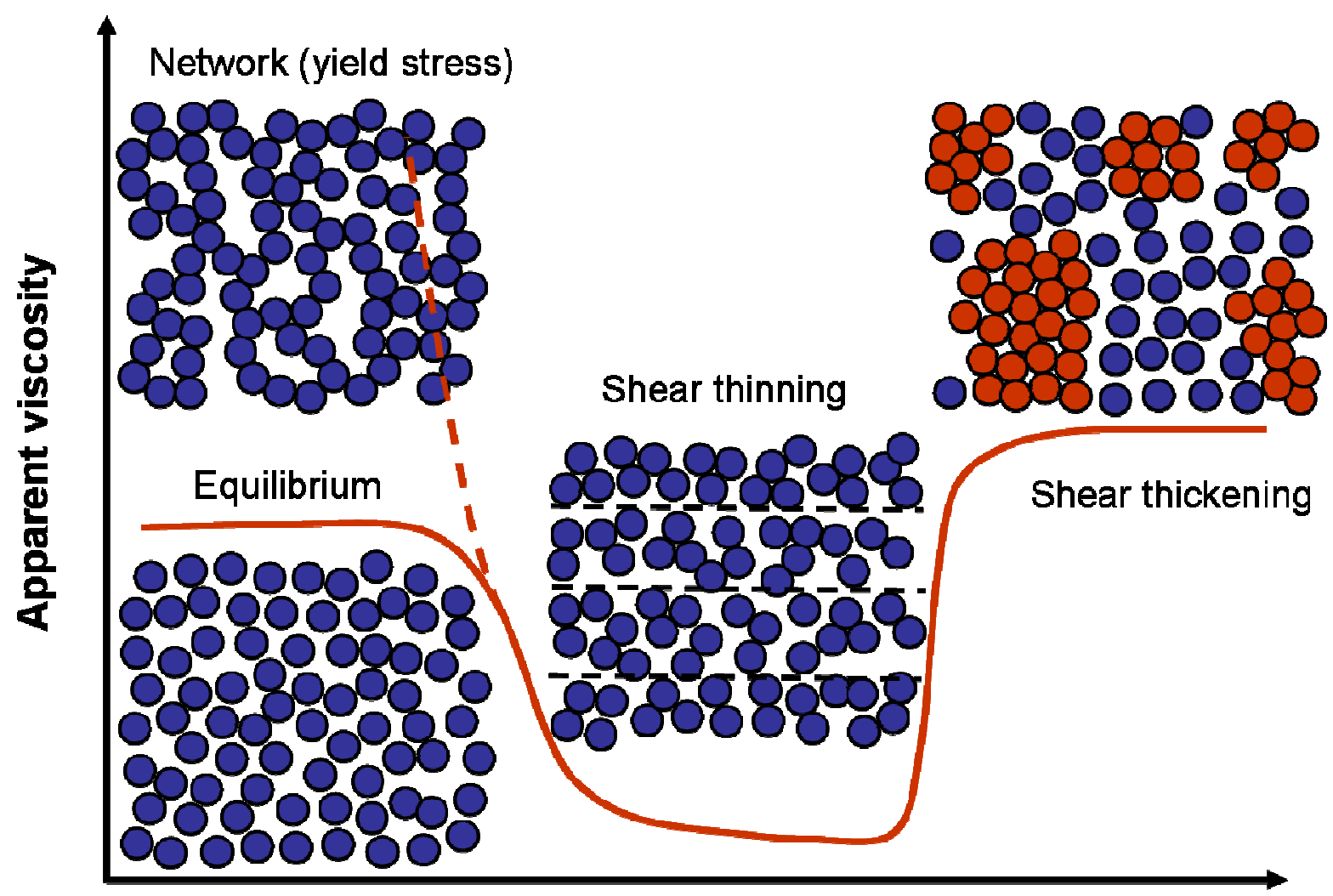

Shear stress or shear rate

Fig. 1 Schematic depicting the effect of suspension microstructure on apparent viscosity, modified from Wagner and Brady. ${ }^{[2]}$ Particles in hydroclusters are shown in red. 


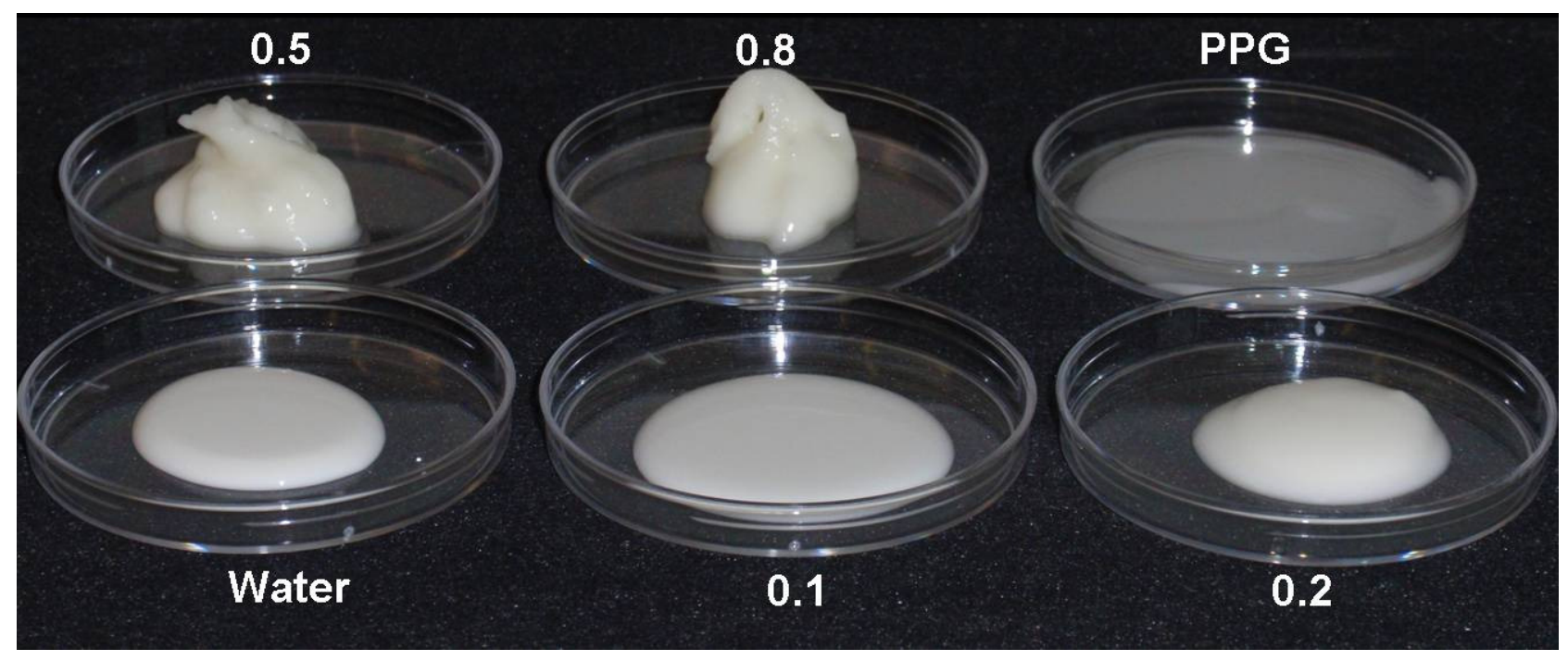

Fig. 2 Physical appearance of $50 \mathrm{wt} \% \mathrm{CS}$ suspensions for different $\phi_{\mathrm{PPG}}$ (indicated). 


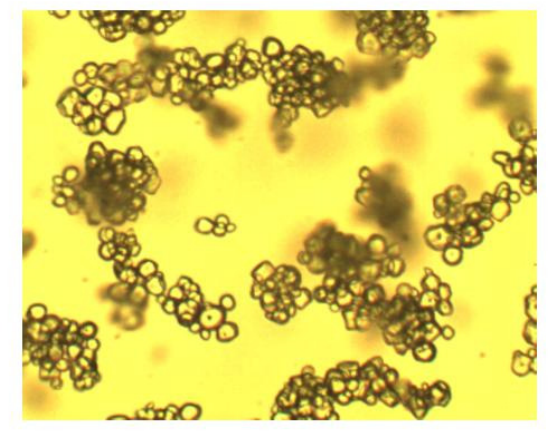

0.5

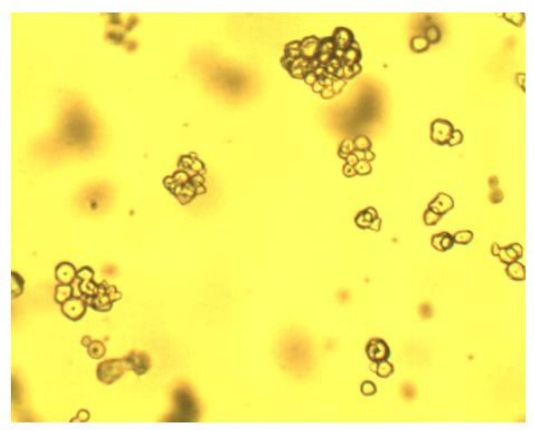

0.8

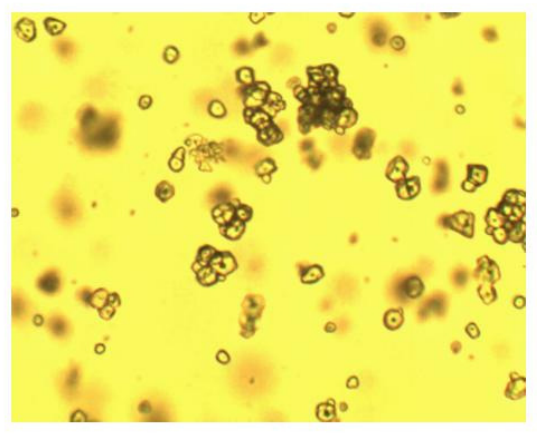

PPG

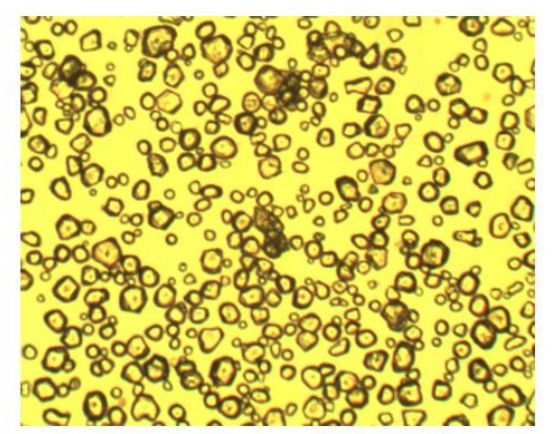

Water

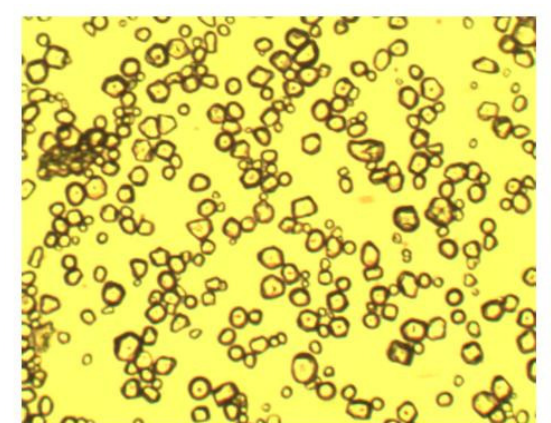

0.1

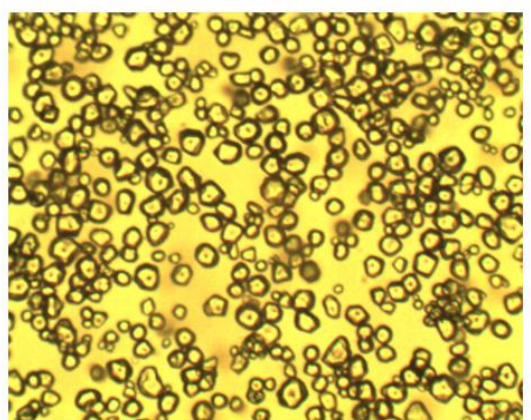

0.2

$50 \mu \mathrm{m}$

Fig. 3 Optical photomicrographs of 1 wt $\%$ CS suspensions for different PPG/water compositions ( $\phi_{\mathrm{PPG}}$ values indicated). 


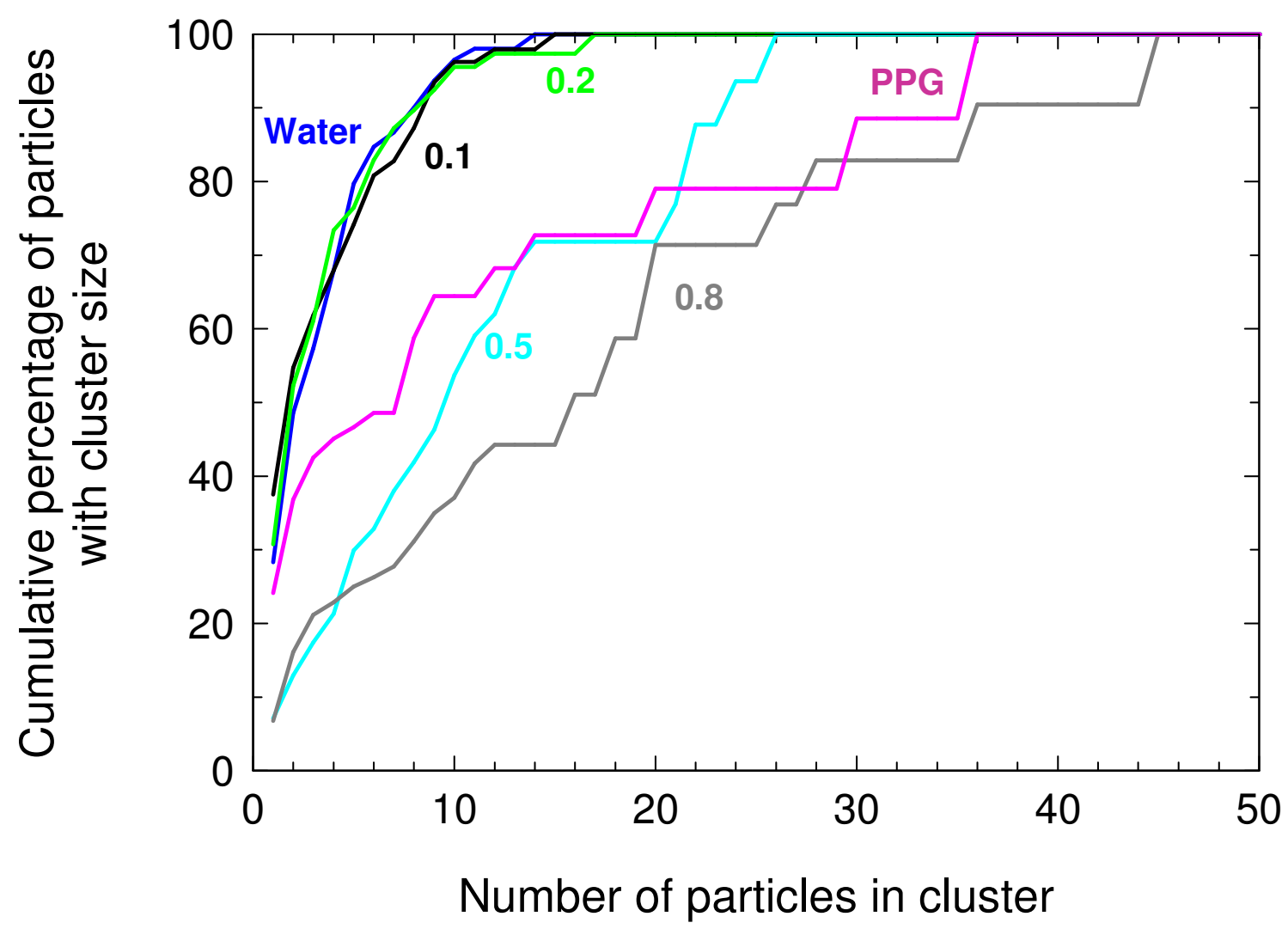

Fig. 4 Particle cluster distribution analysis for different $\phi_{\mathrm{PPG}}$. 

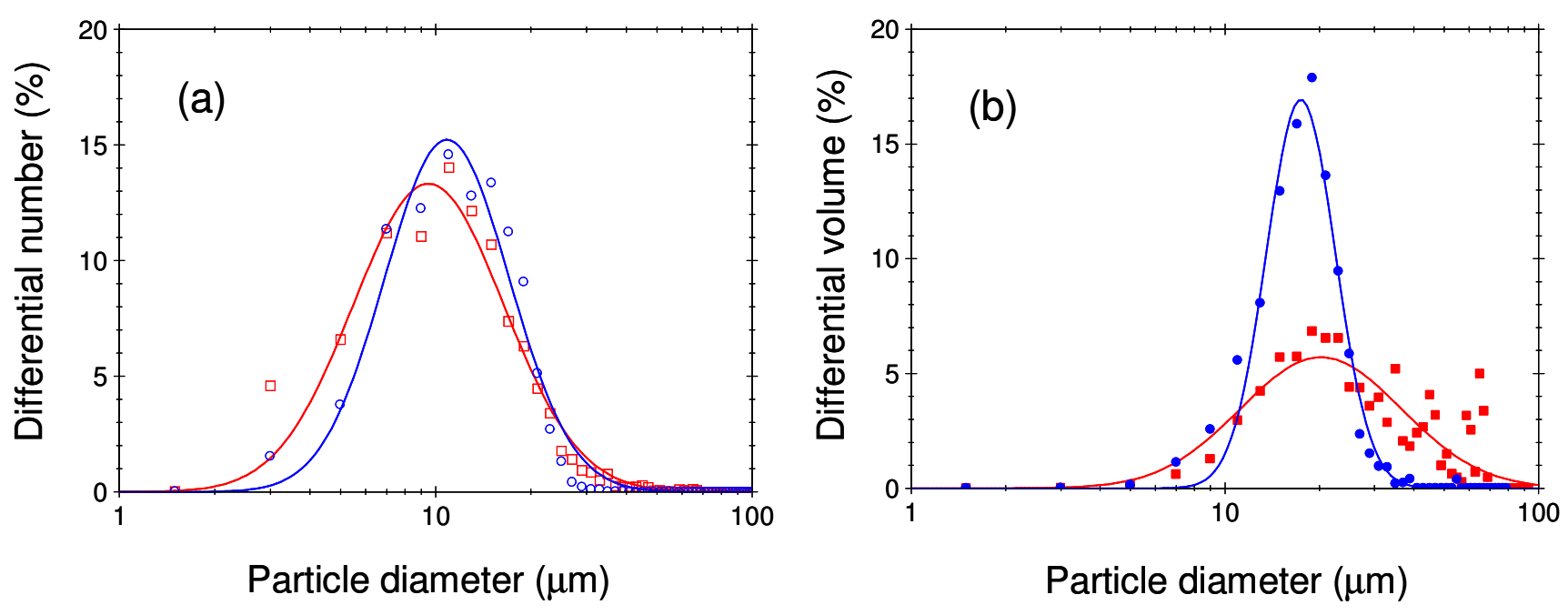

Fig. 5 (a) Number and (b) volume cornstarch particle size distributions in water (blue circles) and PPG (red squares). The curves are log-normal distribution fits. 


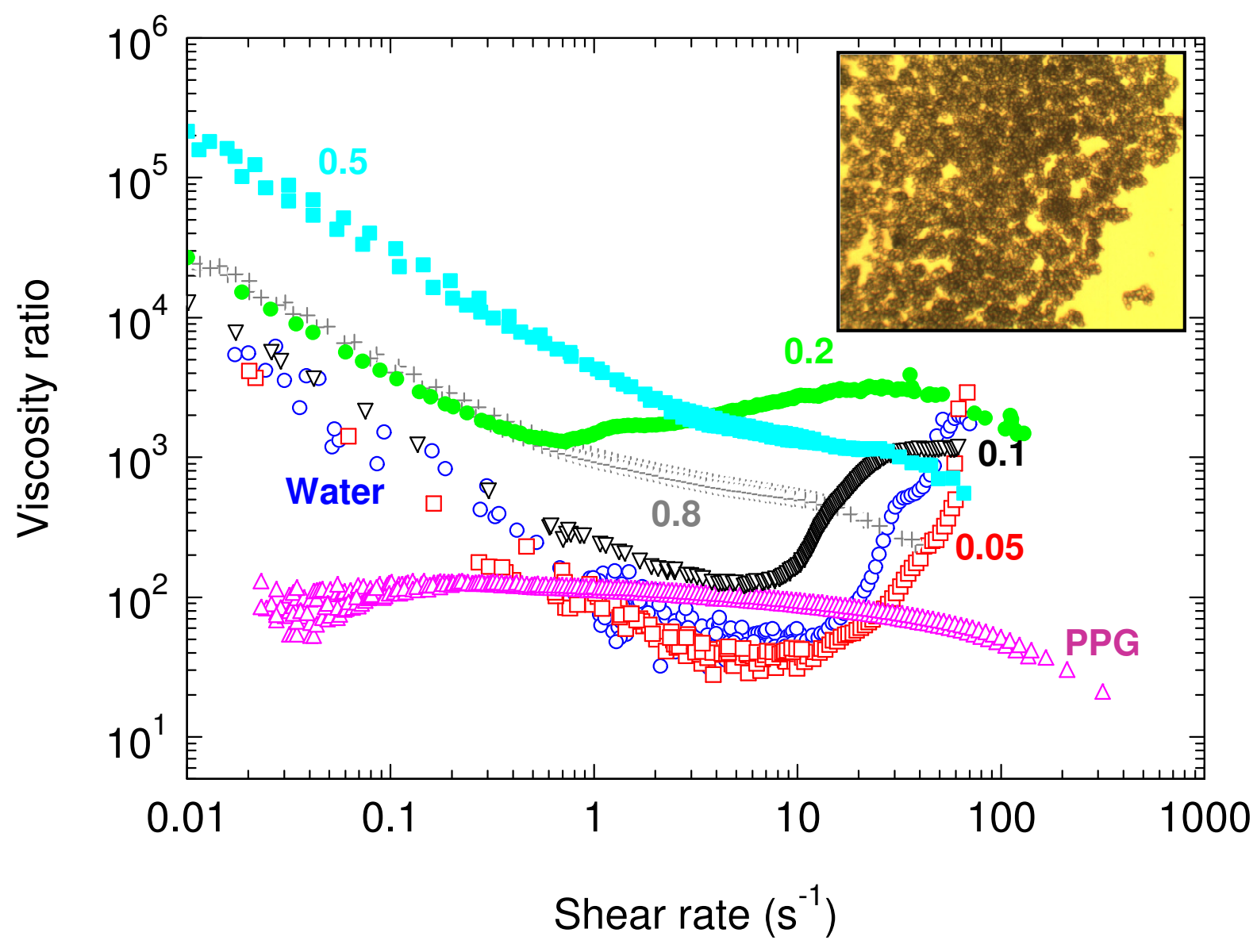

Fig. 6 Steady shear viscosity of 50\% CS suspensions in the indicated PPG/water mixtures. The inset shows the open structure of the $\phi_{\mathrm{PPG}}=0.5$ suspension produced under mild flow conditions. 


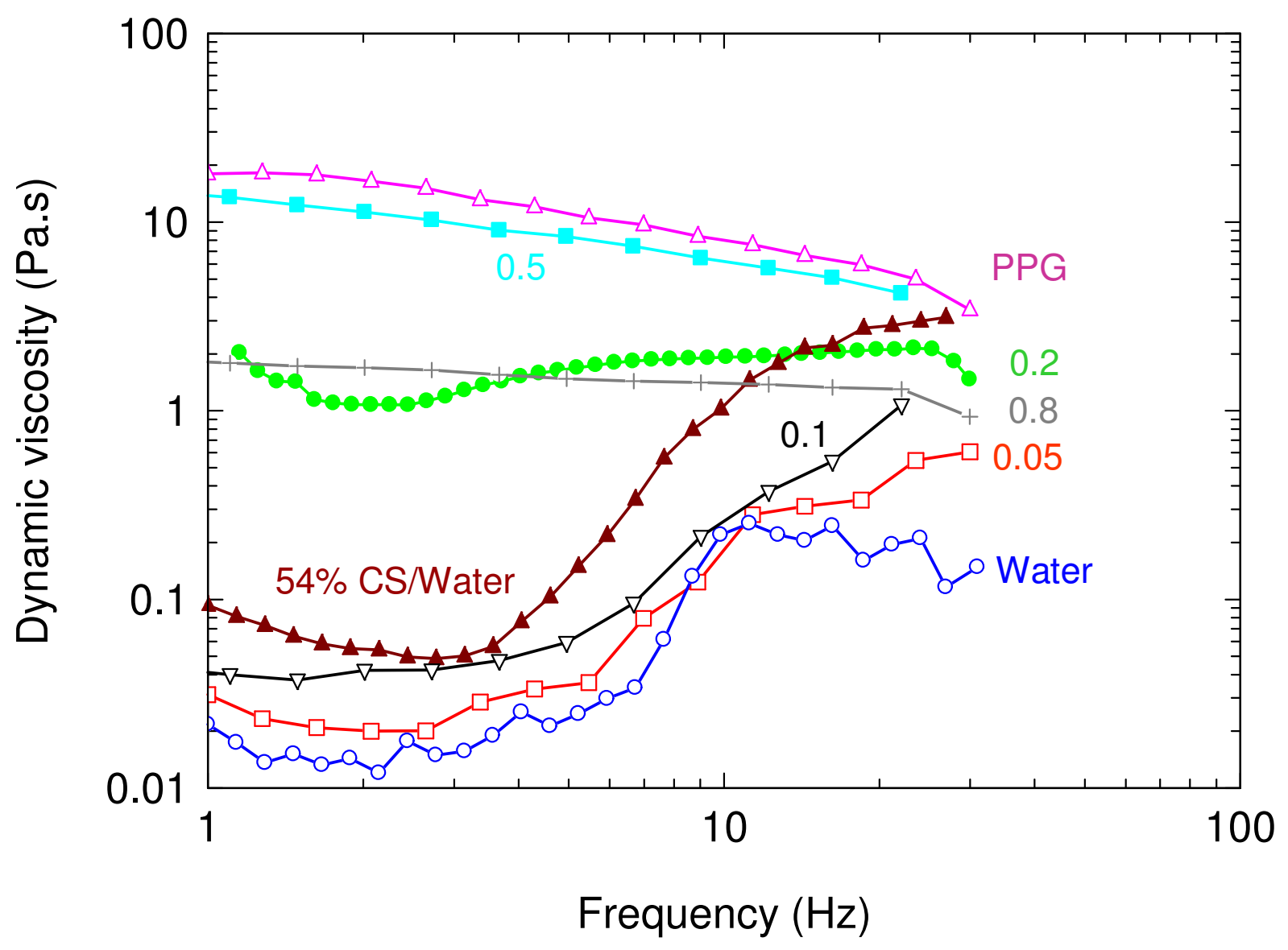

Fig. 7 The effect of oscillation frequency on dynamic viscosity for 50\% CS suspensions in different PPG/water mixtures. For comparison, data for a more concentrated CS suspension in water are also shown. 


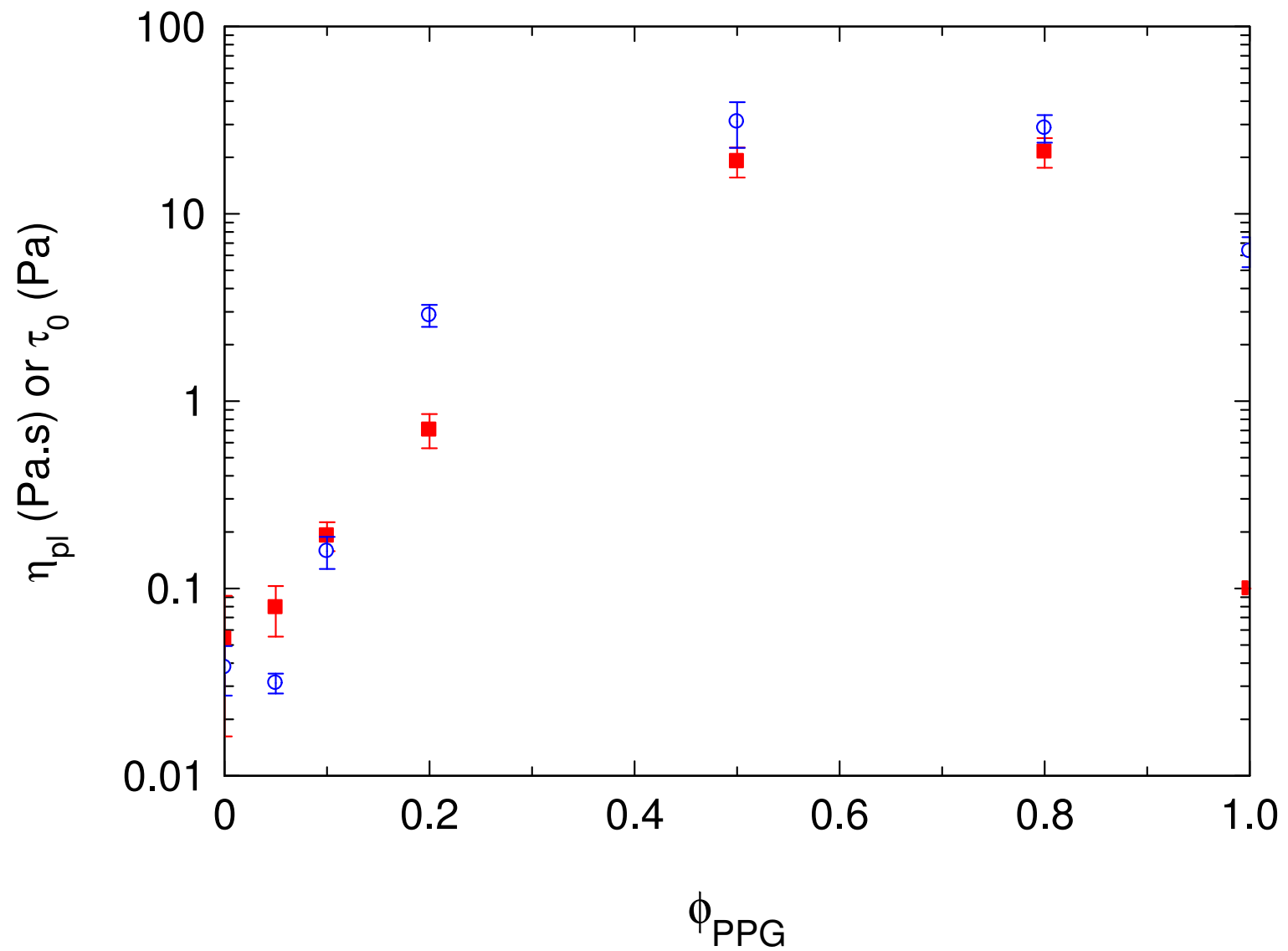

Fig. 8 Plots of the Bingham parameters, plastic viscosity (circles) and yield stress (squares), for $50 \%$ CS suspensions in different PPG/water mixtures. 

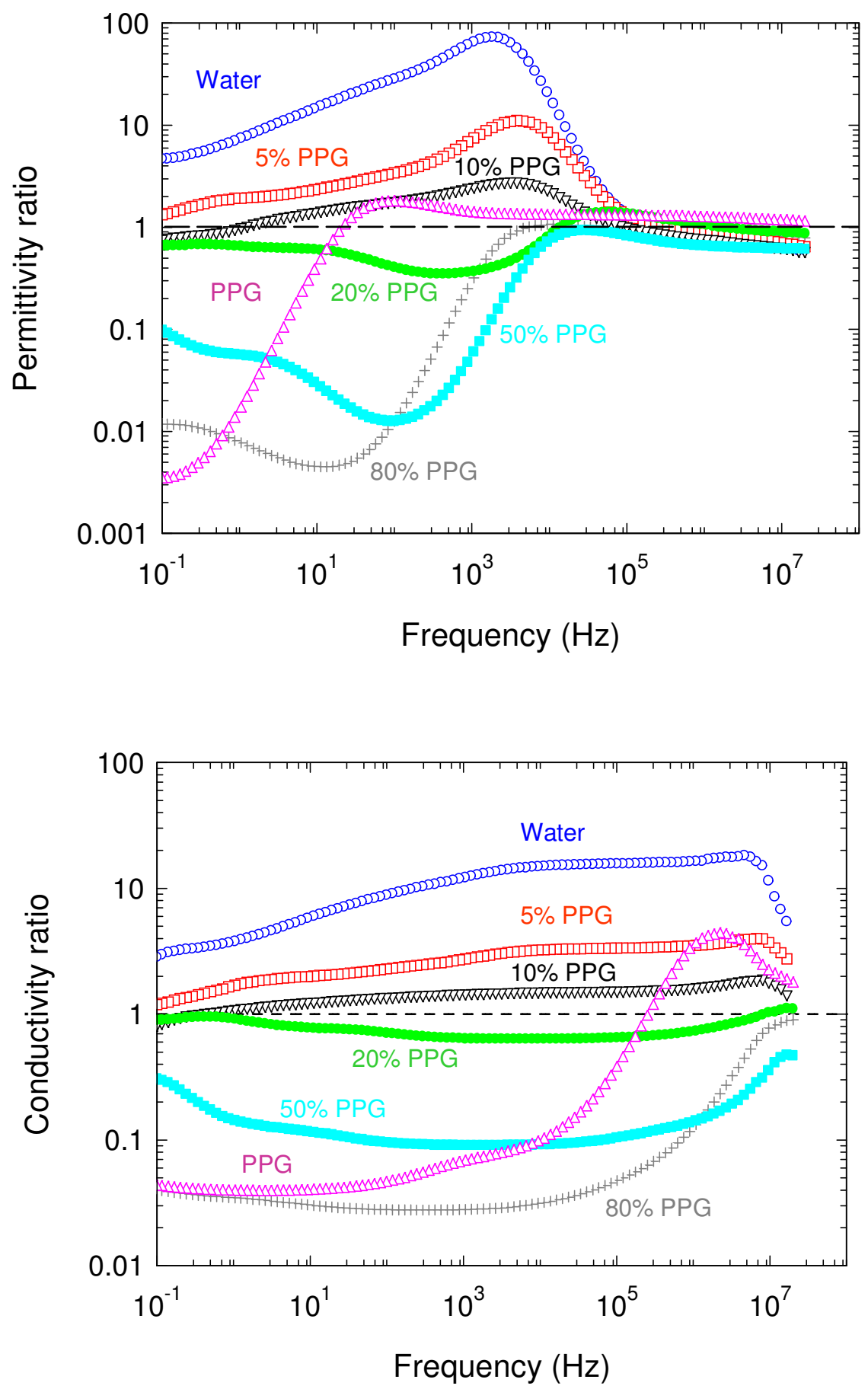

${ }_{10}$ Fig. 9 Permittivity ratio (upper plot) and conductivity ratio (lower plot) spectra for $50 \mathrm{wt} \%$ cornstarch suspensions in different PPG/water mixtures (PPG concentrations indicated). 


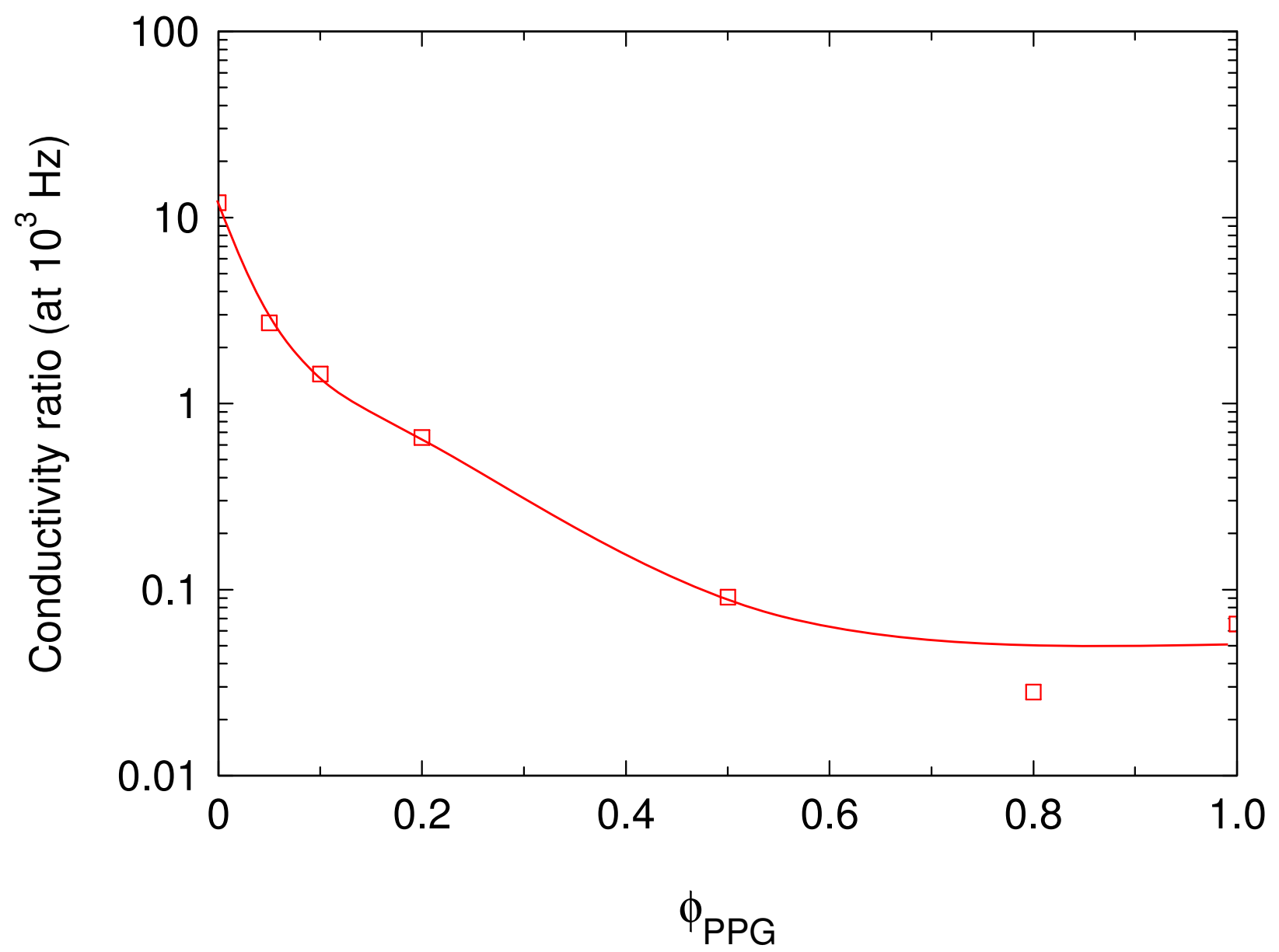

Fig. 10 Effect of PPG/water mixture composition on the conductivity ratio (= suspension conductivity/medium conductivity) at $10^{3} \mathrm{~Hz}$ for $50 \% \mathrm{CS}$ suspensions. The drawn curve is to guide the eye. 


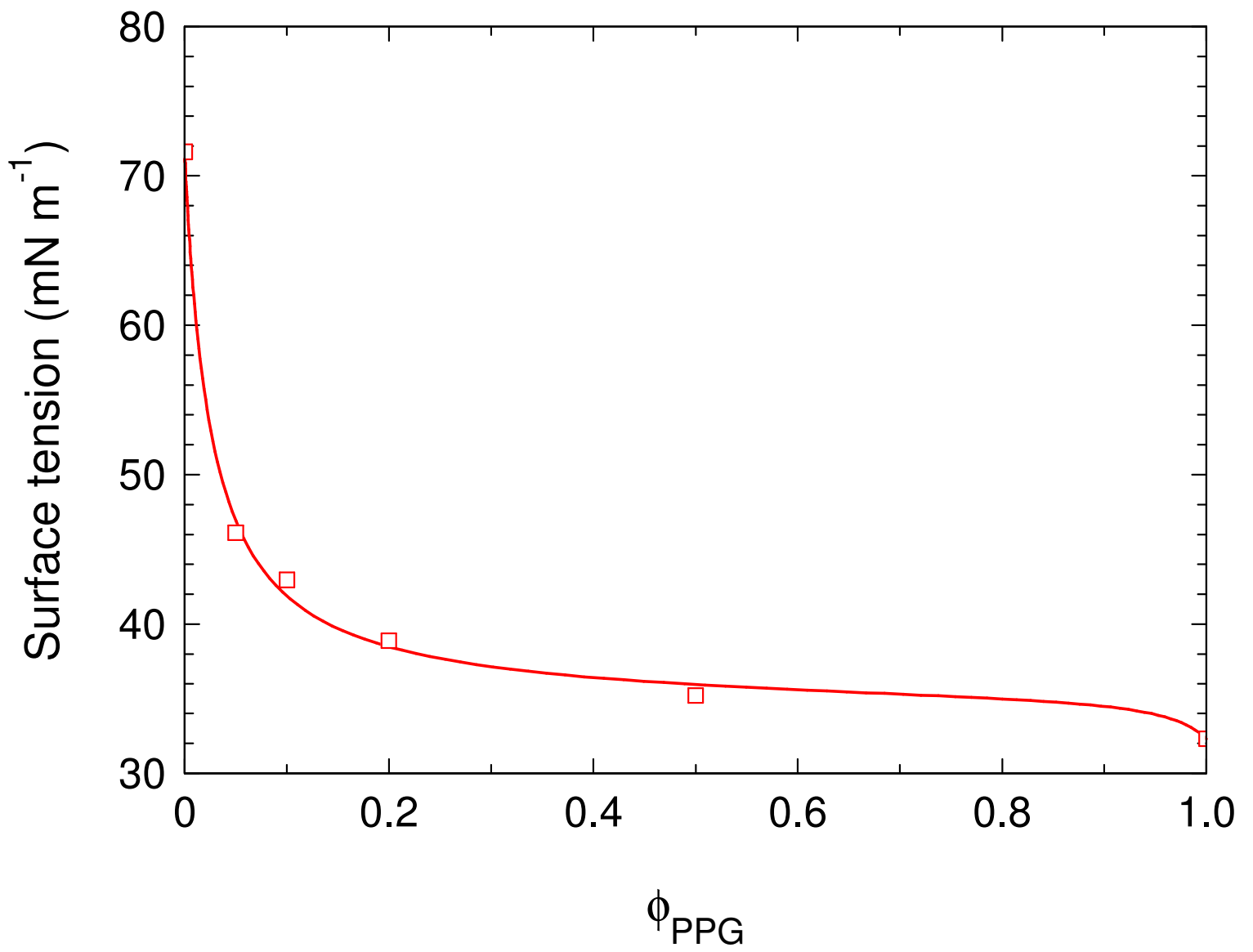

Fig. 11 Effect of PPG mass fraction on surface tension. The curve is derived using eq 4. 


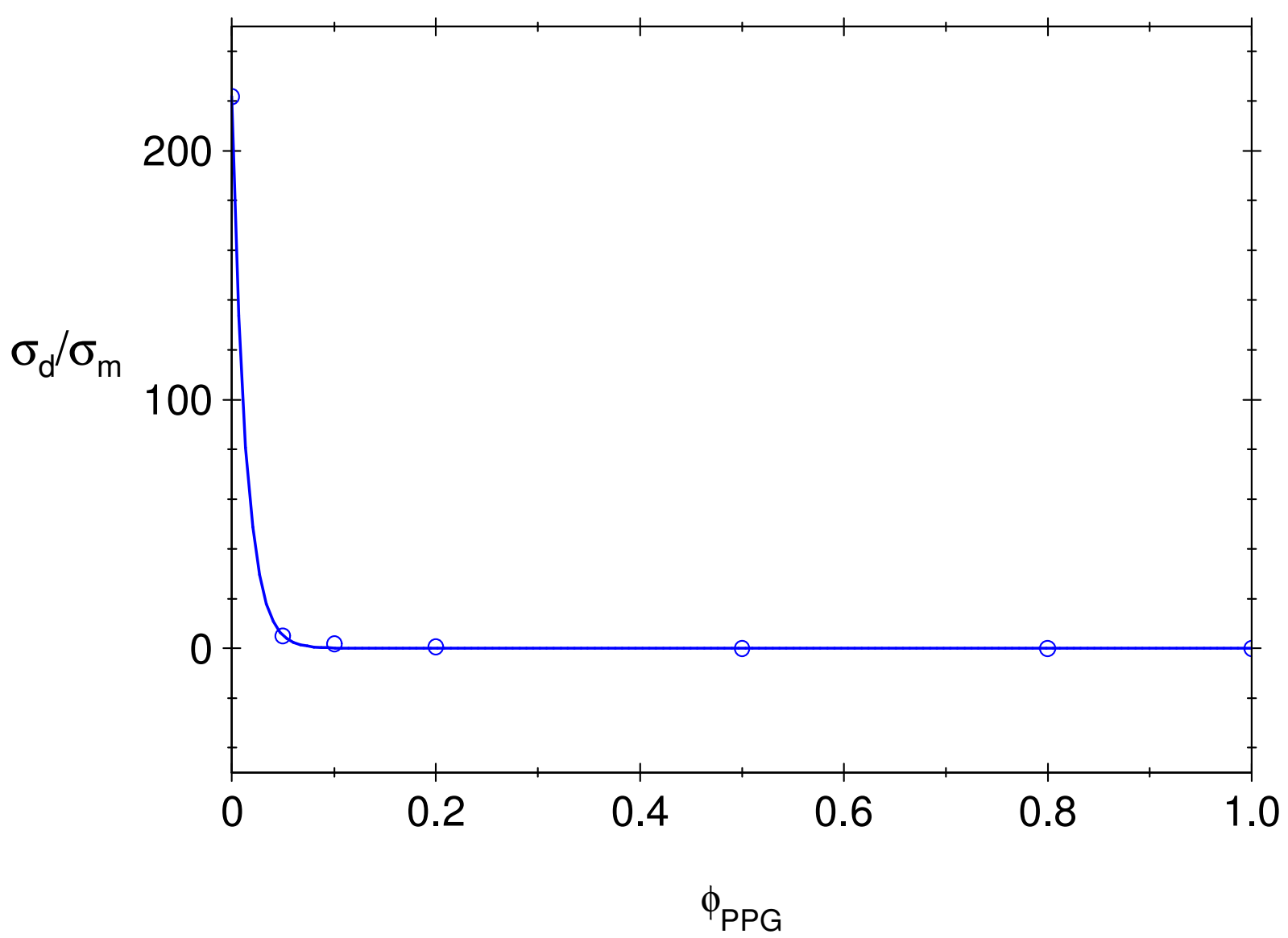

Figure 12. Effect of $\phi_{\mathrm{PPG}}$ on particle/medium conductivity ratio $(\lambda)$. 


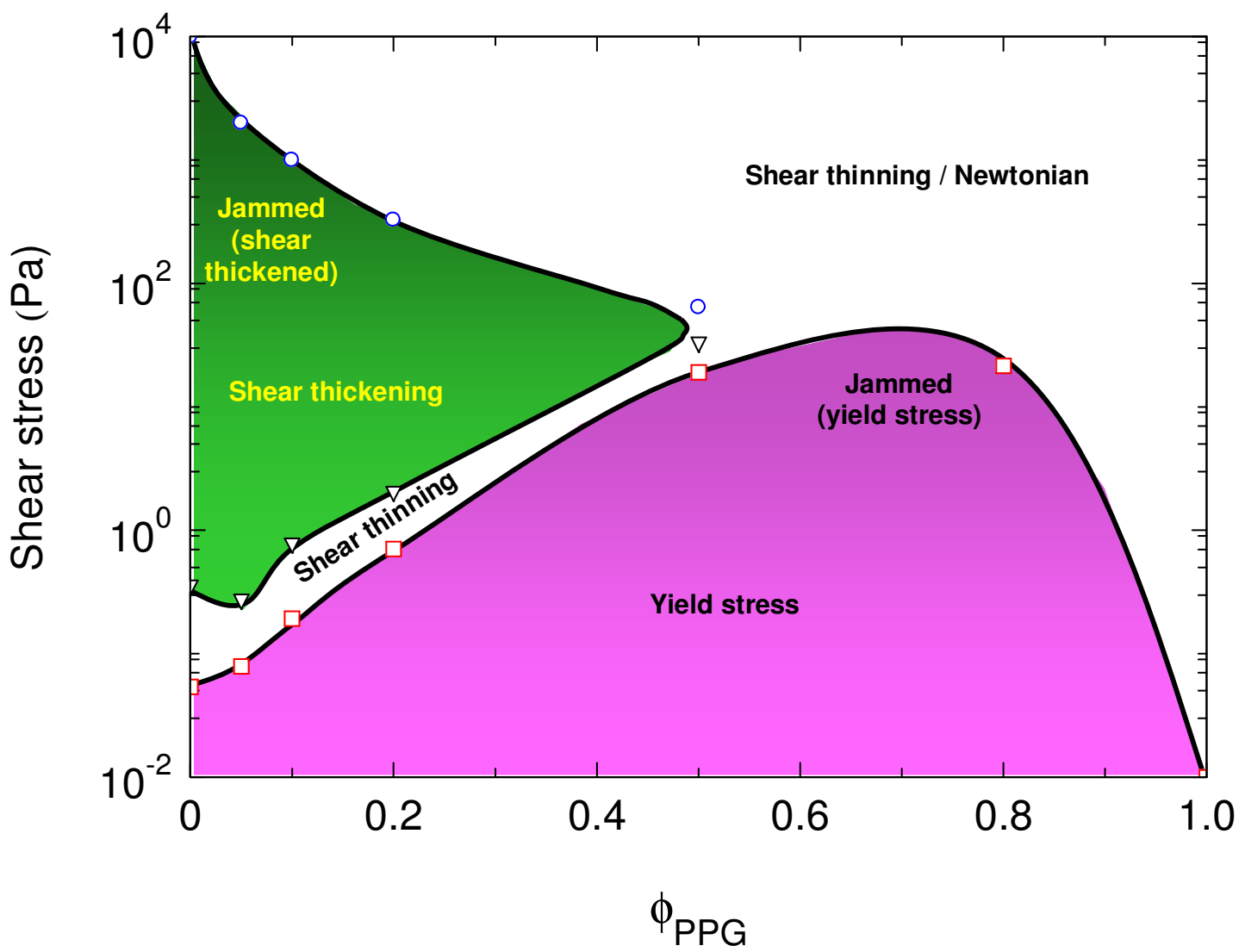

Fig. 13 Non-equilibrium phase diagram defining the different rheological mechanisms for $50 \%$ CS suspensions in PPG/water. 